\title{
Mindfulness-based cognitive therapy in patients with depression: current perspectives
}

This article was published in the following Dove Press journal:

Neuropsychiatric Disease and Treatment

\author{
Meagan B MacKenzie' \\ Kayleigh A Abbott ${ }^{2}$ \\ Nancy L Kocovski ${ }^{2}$ \\ 'Department of Psychology, Ryerson \\ University, Toronto, ON, Canada; \\ ${ }^{2}$ Department of Psychology, Wilfrid \\ Laurier University, Waterloo, ON, \\ Canada
}

\begin{abstract}
Mindfulness-based cognitive therapy (MBCT) was developed to prevent relapse in individuals with depressive disorders. This widely used intervention has garnered considerable attention and a comprehensive review of current trends is warranted. As such, this review provides an overview of efficacy, mechanisms of action, and concludes with a discussion of dissemination. Results provided strong support for the efficacy of MBCT despite some methodological shortcomings in the reviewed literature. With respect to mechanisms of action, specific elements, such as mindfulness, repetitive negative thinking, self-compassion and affect, and cognitive reactivity have emerged as important mechanisms of change. Finally, despite a lack of widespread MBCT availability outside urban areas, research has shown that self-help variations are promising. Combined with findings that teacher competence may not be a significant predictor of treatment outcome, there are important implications for dissemination. Taken together, this review shows that while $\mathrm{MBCT}$ is an effective treatment for depression, continued research in the areas of efficacy, mechanisms of action, and dissemination are recommended.
\end{abstract}

Keywords: mindfulness-based cognitive therapy, MBCT, major depressive disorder, efficacy, mechanisms of action, dissemination

\section{Introduction}

Major depressive disorder (MDD) - characterized by pervasive and persistent low mood and lack of interest in normally pleasurable activities - is one of the most common mental health disorders, with high personal and societal costs. The lifetime prevalence rate of MDD is estimated to be approximately $16 \%$ and has a high recurrence rate, ranging from $35 \%$ in depressed patients from the general population to $85 \%$ in depressed patients from clinical samples. ${ }^{1,2}$ As a result of this prevalent and debilitating disorder, mindfulness-based cognitive therapy (MBCT) was developed to reduce depressive relapse for individuals with recurrent MDD. ${ }^{3}$

MBCT is a group-based intervention that draws from elements of mindfulness-based stress reduction (MBSR) and cognitive behavioral therapy (CBT). ${ }^{4,5}$ Mindfulness is defined as: "the awareness that emerges through paying attention on purpose, in the present moment, and non-judgmentally to things as they are". ${ }^{6} \mathrm{MBCT}$ includes systematic training in mindfulness meditation by drawing on MBSR, such that patients practice mindfulness exercises (eg, body scans) to become more aware of their thoughts, emotions, and body sensations. In addition, MBCT contains elements of CBT, such as psycho-education about the role of cognition in depression and exercises to illustrate how one's thoughts, emotions, and behaviors, induce and maintain depressive symptoms. The combination of mindfulness and CBT used within MBCT is thought to help individuals recognize the automatic activation of dysfunctional cognitive
Correspondence: Nancy L Kocovsk Department of Psychology, Wilfrid Laurier University, 75 University Ave W, Waterloo, ON N2L 3C5, Canada

$\mathrm{Tel}+$ I 5198840710 ext 35I9

Fax +I 5197467605

Email nkocovski@wlu.ca 
processes, such as ruminative and negative thoughts, and to disengage from these dysfunctional processes with the goal of reducing depressive symptoms and future risk of depressive relapse.

\section{Present review}

This review summarizes current research pertaining to the efficacy of MBCT in comparison to treatment-as-usual (TAU; the usual treatment according to accepted standards) and active-control conditions (ACCs) for MDD. This review also examines mechanisms of change for this therapy and current dissemination practices, and in particular how these identified mediators and dissemination strategies relate to treatment outcomes of MDD. This narrative review aims to inform clinical practice and steer future research toward enhanced investigation into efficacy, mechanisms of action, and dissemination practices of $\mathrm{MBCT}$ for the treatment of MDD.

\section{Efficacy of MBCT for depression}

Several systematic reviews and meta-analyses have shown that MBCT, conducted in accordance with the manual, significantly reduces risk of relapse and related symptoms for individuals in depressive remission, and more recently for individuals with current depressive symptomology. ${ }^{7-11}$ Overall, results have shown that MBCT is effective at reducing the rate of depressive relapse along with a reduction of depressive symptoms. The following will overview results from several systematic reviews, meta-analyses, and randomized controlled trials (RCTs), exploring the efficacy of MBCT in relation to TAU groups, waitlist control groups, and ACCs for the outcome variables: depressive relapse and depressive symptoms.

Empirical evidence supporting the use of MBCT to decrease the risk of depressive relapse started with an initial trial conducted by Teasdale et al in $2000 .{ }^{12}$ Teasdale et al found that individuals who were in remission or recovery from major depression had a lower rate of relapse in the MBCT group (40\%) compared to the TAU group (66\%). Several studies have also found that individuals in remission or recovery from depression have significantly lower relapse rates in the MBCT plus medication group compared to a medication-alone group. ${ }^{13,14}$

In addition, MBCT compared to TAU, has been associated with a reduction in depressive and comorbid symptoms and increased quality of life. ${ }^{13,14}$ Further, in 2012, van Aalderen et al found that individuals in the MBCT plus TAU group reported less depressive symptoms, worry and rumination, and increased levels of mindfulness compared to the TAU alone group..$^{15}$ More recent work by Forkman et al showed a significant reduction in self-reported suicidal ideation in the MBCT group for individuals with residual depressive symptoms. ${ }^{16}$

MBCT has also been supported as an effective therapy option. In 2007, Coelho et al conducted a systematic review of four studies that compared MBCT plus TAU to TAU alone. ${ }^{8}$ They concluded that MBCT is a positive addition to TAU for individuals who have experienced three or more depressive episodes. However, this conclusion was qualified given the need for control groups that are better matched to the MBCT program. Clarke et al conducted a systematic review and meta-analysis in 2015 examining the effect of non-pharmacological interventions on depressive relapse prevention and concluded that MBCT along with $\mathrm{CBT}$ and interpersonal psychotherapy were each associated with a significant reduction in relapse compared to controls. ${ }^{7}$ However, the authors suggested that the generalizability of these findings warranted caution, given that MBCT was largely tested following medication and may be limited only to patients who had previously recovered from their depression through pharmacological means. A more recent meta-analysis conducted by Kuyken et al in 2016 analyzed individual patient data from nine published randomized trials of MBCT. ${ }^{9}$ Kuyken et al examined the efficacy of MBCT compared with usual care or active treatment groups for patients from a range of sociodemographic backgrounds. Replicating prior work, results provided evidence that MBCT was associated with a significant reduction in the risk of depressive relapse compared with usual care, with a larger effect being found for individuals with higher levels of depression symptoms at baseline. The latter finding is consistent with recent trials that have shown MBCT is more effective for people whose depressive symptoms fluctuate and/or who report a history of early adversity. ${ }^{14,17,18}$ Kuyken et al also replicated prior work supporting that MBCT does not have differential effects for individuals based on their sex, age, education, or relationship status. ${ }^{9}$

The focus thus far has been on preventing depressive relapse. Depressive symptoms and other related outcomes have also been investigated. In 2014, Strauss et al conducted a meta-analysis that evaluated 12 studies investigating mindfulness-based interventions for individuals with current anxiety or major depression. MBCT significantly reduced primary symptom severity for those with current depression, compared to control conditions. ${ }^{11}$ More recently, PeresteloPerez et al conducted a systematic review and meta-analysis 
in 2017 to evaluate the effect of mindfulness-based interventions on ruminative thoughts in individuals with current or past depressive disorders. ${ }^{10}$ Results of 11 studies showed that MBCT compared to usual care produced a significant reduction in rumination, independent of the number of past depressive episodes.

Although MBCT has thus far been shown to be effective, one limitation of the aforementioned studies is the absence of an active control group. To address this limitation, recent studies have compared MBCT to ACCs, exhibiting mixed findings for significant group difference on depressive relapse and related symptoms.

Trials including depressive relapse as a primary outcome variable have thus far found no difference between MBCT and ACCs. For instance, in a 2014 trial, Williams et al compared MBCT to cognitive psychological education (all elements of MBCT except for experiential mindfulness) and TAU in an RCT with individuals who had three or more episodes of recurrent depression. ${ }^{18}$ MBCT was only a more effective treatment for relapse prevention in individuals who experienced childhood trauma, but when examining the whole sample MBCT did not show a significant advantage to the ACC or TAU. Further, in a study conducted by Shallcross et al in 2015, MBCT did not differ from the ACC on variables such as rate of depression relapse, symptom reduction, and life satisfaction. ${ }^{19}$ Conversely, in 2014, Meadows et al found more positive results when comparing MBCT to ACCs. ${ }^{20}$ Meadows et al found that MBCT combined with depression-relapse active monitoring was superior to depression-relapse active monitoring alone in reducing depressive relapse over a 2-year follow-up for individuals not currently experiencing depressive symptoms but with a history of three or more depressive episodes.

Mixed outcomes for the effectiveness of MBCT compared to ACC have also been found for trials measuring depressive symptoms as the primary outcome variable. In a recent RCT conducted by Eisendrath et al in 2016, MBCT was compared to a health-enhancement program, which included physical fitness, music therapy, and nutritional education, in individuals with treatment-resistant depression. ${ }^{21}$ MBCT was significantly associated with reduced depressionsymptom severity compared to the health-enhancement program. Further, in 2015, Chiesa et al used an RCT to compare MBCT to a psycho-education program in a small sample of 43 patients with depressive disorder who had not achieved remission following the use of antidepressants. ${ }^{22}$ Compared to the psycho-education program, individuals in the MBCT group showed significant improvements in depressive symptoms, as well as on measures of mindfulness and quality of life. Conversely, in 2011, Manicavasgar et al found no group differences in outcomes for the treatment of depression between an 8-week MBCT and CBT group for individuals with a current depressive disorder. ${ }^{23}$

A more recent meta-analysis conducted by PeresteloPerez et al compared the effect of MBCT to ACCs on rumination for individuals with a depressive disorder. ${ }^{10}$ Using three studies, results indicated that MBCT appeared as effective at reducing rumination as other treatments such as medication, CBT, and behavioral activation. However, authors recommended caution when interpreting these results given the low number of studies included.

Overall, these analyses provide strong evidence for the effectiveness of MBCT in individuals who are in remission or currently experiencing a depressive episode when compared to TAU; however, discrepant findings are present when compared to ACCs. In addition, the conclusions of current research are constrained by several limitations, such as the heterogeneity of the measures used, the need for more appropriate control groups, and the need for more long-term follow up data. Given the variation in findings and limitations it is important to continue to evaluate the efficacy of MBCT, especially compared to appropriate ACCs.

\section{Mechanisms of change in MBCT}

In addition to evaluating MBCT outcomes, it is important to clarify the mechanisms of action of this intervention. Kazdin has provided a useful resource for conducting research with the goal of demonstrating mediators and mechanisms of change for psychotherapy. He defines mechanisms as "the processes or events that are responsible for change" and underscores the need for greater attention to these lines of research. ${ }^{24}$ Perhaps increased collaboration among clinical and neuroscience practitioners may help to shed some light on the neural mechanisms underpinning MBCT. van der Velden and Roepstorff have called for such alliances to assist in clarifying the processes by which MBCT works. ${ }^{25}$ To date, there does not appear to be such a collaboration, but further understanding of these and other underlying processes will certainly allow for optimization of this treatment modality, resulting in enhanced treatment efficacy.

The available RCTs of MBCT for depression provide strong support in favor of MBCT although some have clear limitations along with mixed outcomes. Given some equivocal findings, the examination of mechanisms of action is preliminary at this point in time, and further research is necessary to strengthen this research area. Despite a small 
body of rigorous evaluations of mechanisms of change within mindfulness-based interventions, recent work has been published. To date, three systematic reviews have been conducted to identify and examine mechanisms of action in mindfulness-based therapies. Gu et al conducted a systematic review and meta-analysis evaluating mechanisms of action underlying mindfulness-based interventions for psychiatric disorders. ${ }^{26}$ Rather than including only studies with participants who were diagnosed with MDD, this review included any mental health outcomes. The results of their analyses showed that both mindfulness and repetitive negative thinking mediated the effects of mindfulness-based interventions for several outcomes including anxiety, depression, general psychopathology, stress, and negative affect.

More recently, Alsubaie et al conducted a systematic review of mechanisms of action in mindfulness-based interventions for individuals with either physical or psychological health conditions. ${ }^{27}$ Studies were included if they were trials designed to examine mechanisms of change in MBCT/ MBSR in adults with either physical or psychological conditions. They reviewed six studies in their section related to mechanisms of action in mindfulness-based interventions for depression..$^{10,28-32}$ Results showed that positive treatment outcomes were mediated by significant increases in mindfulness, rumination, affect, self-compassion, reactivity, and cognitive function. In a 2015 systematic review specific to MDD, van der Velden et al reviewed 23 clinical trials of MBCT for recurrent MDD to evaluate mechanisms of change. ${ }^{25}$ Studies were included if they examined mechanisms in MBCT for MDD, had adult participants, and MBCT was conducted in accordance with the manualized procedures. The results showed that in 12 of the 23 included studies, MBCT outcome was associated with or predicted by changes in mindfulness, rumination, worry, self-compassion, decentering, and/or meta-awareness. Given the overlap among mechanisms identified in the reviews, several of the predominant mechanisms of change will be discussed.

\section{Mindfulness}

Mindfulness is most often defined using Kabat-Zinn's description of nonjudgmental present moment attention. ${ }^{33}$ Perhaps the most important inquiry is whether positive outcomes in MBCT are due to mindfulness being a significant mediator. Both Alsubaie et al and Gu et al identified RCTs in their systematic reviews showing that MBCT reduces depressive symptoms ${ }^{26,27}$ Moreover, these effects appear to be mediated by increases in mindfulness, acceptance, and curiosity even after taking into account heterogeneity of measurement, samples, and outcome assessment. ${ }^{10,28-32,34,35}$

\section{Repetitive negative thinking}

Repetitive negative thinking involves thinking about negative experiences and is implicated in numerous psychopathologies. This cognitive pattern is difficult to disengage from and is often conceptualized as worry and rumination; these two cognitive patterns differ in their temporal orientation. Worry is typically future-oriented and rumination is past-oriented, and there is significant overlap among these constructs. ${ }^{36,37}$ Rumination is a way of coping with distress and refers to repetitively thinking about one's unpleasant thoughts and feelings and their potential causes and consequences..$^{38}$ Rumination can be assessed as a unidimensional construct and/ or as separate subscales including reflection and brooding. ${ }^{39}$ Across the systematic reviews, three MBCT RCTs have shown that a decrease in depressive symptoms was mediated by rumination, worry, and/or brooding. ${ }^{10,28,30}$ The reflective, pondering component of rumination (assessed as reflection in self-report surveys) was not related. ${ }^{30}$

\section{Self-compassion and positive affect}

Only one RCT has been identified which assessed selfcompassion as a possible mediator. Kuyken et al's study supported self-compassion as a significant mediator of the positive effects of MBCT for individuals with recurrent depression and more than three depressive episodes. Specifically, learning self-compassion skills had a mediating role in MBCT for depression such that an increase in selfcompassion led to treatment gains over a 15-month follow-up period. ${ }^{29}$ Both positive and negative affect have been shown to have mediating roles in the relationship between MBCT and depression. Two RCTs have indicated that increased positive affect, including pleasant activities and rewarding experiences and a decrease in negative affect have both been implicated in depression reduction. ${ }^{28,40}$

\section{Cognitive and emotional reactivity}

Two RCTs included cognitive and emotional reactivity as potential mediators of the effects of MBCT on depressive symptomatology in their analyses. ${ }^{29,35}$ These constructs refer to the diathesis-stress model of psychopathology; specifically, the extent to which a state of distress combined with stress reactivates negative cognitions and affect, putting individuals at risk of a subsequent depressive episode.$^{41}$ For individuals with recurrent depressive episodes, reactivity was a significant mediator among MBCT participation and depressive symptoms.

Taken together, the results of these three systematic reviews, and an examination of their included RCTs yields strong preliminary evidence for several mechanisms of action 
in MBCT for individuals with MDD. These mechanisms include mindfulness, repetitive negative thinking, affect and self-compassion, and cognitive and emotional reactivity. These mechanisms appear to be fundamentally consistent with the theoretical foundations of mindfulness-based interventions, including MBCT. The main premise of MBCT is that the development of mindfulness skills can lead to an increase in nonjudgmental acceptance of symptoms, thereby leading to a reduction of such symptoms. ${ }^{3,42}$ These initial findings likewise support the idea that a reduction in worry and rumination allows for disengagement with symptoms, leading to treatment gains. Next steps for this line of inquiry should include more in-depth identification and analyses of mechanisms, as well as replication of the few robust studies included here.

\section{Dissemination of MBCT}

Access to MBCT remains a key challenge. Similar to other empirically supported treatments, the availability of MBCT for the general public is limited, especially outside of large cities. Positive developments in the area of dissemination include the availability of self-help workbooks. ${ }^{6,43}$ There is empirical evidence supporting the effectiveness of the Williams and Penman (2011) workbook in a sample of students experiencing low mood, stress or anxiety. ${ }^{44}$ There are also internet-based approaches, including Mindful Mood Balance, for which there is data supporting efficacy in the reduction of depressive symptoms. ${ }^{45-47}$ Data supporting the use of web-based and other self-help methods are promising for more widespread access to MBCT. Further, studies evaluating the benefits of home practice when MBCT is delivered in its original group format have found a small association between the amount of home formal meditation practice and outcome. ${ }^{48}$ Therefore, what the MBCT participants are doing at home matters in terms of the benefit they gain from MBCT. Taken together, these studies build support for the ability to deliver MBCT outside of a traditional group setting with formally trained MBCT teachers.

The importance of being able to access MBCT outside of the traditional group setting is particularly important once the level of training of MBCT teachers is considered. MBCT teachers are required to undergo significant professional training and to have a personal meditation practice. Segal et al outline a training pathway that includes three phases. ${ }^{49}$ The first phase focuses on preparation including readings, teacher training attendance, and residential experiential retreats. The second phase involves co-facilitating two MBCT classes with an experienced teacher and ongoing supervision. The final phase focuses on becoming a proficient MBCT teacher through ongoing mindfulness practice, supervision, and training. These requirements emphasize competence at each phase and imply that a high level of competence is required to become an effective MBCT teacher. The importance of therapist competence in the delivery of MBCT is a key empirical question.

Therapist competence can be evaluated using the Mindfulness-Based Interventions: Teaching Assessment Criteria (MBI:TAC).$^{50}$ The MBI:TAC was developed in the UK and consists of six domains which can each be rated on a scale of one (incompetent) to six (advanced). Examples of domains include embodiment of mindfulness and conveying course themes. The six domains can be averaged for a single competence score. The MBI:TAC is used in the training pathway previously described. ${ }^{49}$ However, to date, only one study has empirically evaluated the importance of therapist competence in the delivery of MBCT.

Using the MBI:TAC, Huijbers et al evaluated the impact of teacher competence on the delivery of MBCT at 12 locations in the Netherlands. ${ }^{51}$ Patients were all in remission from recurrent depression and had taken antidepressant medication. Two sessions were randomly selected for viewing from each of 15 teachers and rated by two independent raters. The final average score for the teachers in this sample was in-between "advanced beginner" and "competent", with no teachers rated at either extreme (ie, none were rated as incompetent, and none were rated as advanced). They demonstrated at least moderate agreement between their raters for each domain, supporting the reliability of the MBI:TAC.

Surprisingly, contrary to their hypotheses, teacher competence was not significantly associated with any of the key outcome variables, including reduction of depressive symptoms. Additionally, competence was not significantly related to any mechanisms of change or attendance. Recall that no therapists were rated as incompetent. Perhaps attaining a minimal level of competence is the key factor in the delivery of MBCT. However, this was the first study to empirically evaluate the relationship between therapist competence and outcome, and it was limited in scope considering the homogeneity of the sample. Notwithstanding these limitations, as well as the necessity for future research on competence with more diverse samples, this study calls into question the rigorous requirements involved in becoming an MBCT teacher, especially when one considers the data supporting the delivery of MBCT via self-help and web-based methods. If research continues to support that the level of therapist competence does not influence attendance, outcome or process, and that it is possible to deliver MBCT in the absence of a well-trained teacher (in the case of self-help methods), 
perhaps an even stronger focus on non-traditional methods aimed at greater access will be warranted.

\section{Conclusion}

MBCT was developed to reduce depressive relapse in individuals with MDD. Empirical evidence including systematic reviews of RCTs has indicated that MBCT is an effective intervention in this context. Despite strong support in its favor, this body of research is not without its limitations. Notably, there are mixed outcomes when MBCT is compared to ACCs. Other limitations include systematic reviews of few studies and possible issues of pharmacological interventions as confounding variables. Future research would benefit by addressing these weaknesses and including long-term follow-up data or longitudinal studies.

The identification and examination of mechanisms of change has been a fruitful area of research in the last 5-10 years. An increasing number of published studies have shown that there appear to be specific active ingredients in MBCT that are leading to treatment gains. Mindfulness, one such active ingredient, certainly warrants continued study as our knowledge of different facets of the meditative experience develops. ${ }^{52}$

Additionally, there have been some promising results in the area of dissemination, although research on teacher competency is in its infancy, with only one published study to date which did not find an association between competency and outcome. Future research on therapist competency is warranted. However, there is growing evidence in support of selfhelp MBCT workbooks and internet-based protocols. These workbooks will assist in addressing some of the barriers to access for individuals outside large cities or who have other limitations that prohibit them from accessing conventional treatment approaches. Finally, given that there is a lack of diverse research in this area, more work investigating the cross-cultural aspects of MBCT would be beneficial to the consideration of MBCT dissemination.

\section{Disclosure}

The authors report no conflicts of interest in this work.

\section{References}

1. Kessler RC, Aguilar-Gaxiola S, Alonso J, et al. The global burden of mental disorders: an update from the WHO World Mental Health (WMH) surveys. Epidemiol Psichiatr Soc. 2009;18(1):23-33.

2. Hardeveld F, Spijker J, De Graaf R, Nolen WA, Beekman AT. Prevalence and predictors of recurrence of major depressive disorder in the adult population. Acta Psychiatr Scand. 2010;122(3):184-191.

3. Segal ZV, Williams JM, Teasdale JD. Mindfulness-Based Cognitive Therapy for Depression: A New Approach to Relapse Prevention. New York: Guilford Press; 2002.
4. Kabat-Zinn J. Full Catastrophe Living: Using the Wisdom of Your Body and Mind to Face Stress, Pain and Illness. New York: Delacourt; 1990.

5. Beck AT, Rush AJ, Shaw BF, Emery G. Cognitive Therapy of Depression. New York: Guilford Press; 1979.

6. Williams JM, Teasdale JD, Segal ZV, Kabat-Zinn J. The Mindful Way through Depression: Freeing Yourself from Chronic Unhappiness. New York: Guilford Press; 2007.

7. Clarke K, Mayo-Wilson E, Kenny J, Pilling S. Can non-pharmacological interventions prevent relapse in adults who have recovered from depression? A systematic review and meta-analysis of randomised controlled trials. Clin Psychol Rev. 2015;39:58-70.

8. Coelho HF, Canter PH, Ernst E. Mindfulness-based cognitive therapy: evaluating current evidence and informing future research. J Consult Clin Psychol. 2007;75(6):1000-1005.

9. Kuyken W, Warren FC, Taylor RS, et al. Efficacy of mindfulness-based cognitive therapy in prevention of depressive relapse: an individual patient data meta-analysis from randomized trials. JAMA Psychiatry. 2016;73(6):565-574.

10. Perestelo-Perez L, Barraca J, Peñate W, Rivero-Santana A, AlvarezPerez Y. Mindfulness-based interventions for the treatment of depressive rumination: Systematic review and meta-analysis. Int J Clin Health Psychol. 2017;17(3):282-295.

11. Strauss C, Cavanagh K, Oliver A, Pettman D. Mindfulness-based interventions for people diagnosed with a current episode of an anxiety or depressive disorder: a meta-analysis of randomised controlled trials. PLoS One. 2014;9(4):e96110.

12. Teasdale JD, Segal ZV, Williams JM, et al. Prevention of relapse/ recurrence in major depression by mindfulness-based cognitive therapy. $J$ Consult Clin Psychol. 2000;68(4):615-623.

13. Kuyken W, Byford S, Taylor RS, et al. Mindfulness-based cognitive therapy to prevent relapse in recurrent depression. J Consult Clin Psychol. 2008;76(6):966-978.

14. Segal ZV, Bieling P, Young T, et al. Antidepressant monotherapy vs sequential pharmacotherapy and mindfulness-based cognitive therapy, or placebo, for relapse prophylaxis in recurrent depression. Arch Gen Psychiatry. 2010;67(12):1256-1264.

15. Van Aalderen JR, Donders AR, Giommi F, et al. The efficacy of mindfulness-based cognitive therapy in recurrent depressed patients with and without a current depressive episode: a randomized controlled trial. Psychol Med. 2012;42(5):989-1001.

16. Forkmann T, Wichers M, Geschwind N, et al. Effects of mindfulnessbased cognitive therapy on self-reported suicidal ideation: results from a randomised controlled trial in patients with residual depressive symptoms. Compr Psychiatry. 2014;55(8):1883-1890.

17. Kuyken W, Hayes R, Barrett B, et al. Effectiveness and cost-effectiveness of mindfulness-based cognitive therapy compared with maintenance antidepressant treatment in the prevention of depressive relapse or recurrence (PREVENT): a randomised controlled trial. Lancet. 2015;386(9988):63-73.

18. Williams JM, Crane C, Barnhofer T, et al. Mindfulness-based cognitive therapy for preventing relapse in recurrent depression: A randomized dismantling trial. J Consult Clin Psychol. 2014;82(2):275-286.

19. Shallcross AJ, Gross JJ, Visvanathan PD, et al. Relapse prevention in major depressive disorder: mindfulness-based cognitive therapy versus an active control condition. J Consult Clin Psychol. 2015;83(5):964-975.

20. Meadows GN, Shawyer F, Enticott JC, et al. Mindfulness-based cognitive therapy for recurrent depression: a translational research study with 2-year follow-up. Aust N Z J Psychiatry. 2014;48(8):743-755.

21. Eisendrath SJ, Gillung E, Delucchi KL, et al. A randomized controlled trial of mindfulness-based cognitive therapy for treatment-resistant depression. Psychother Psychosom. 2016;85(2):99-110.

22. Chiesa A, Castagner V, Andrisano C, et al. Mindfulness-based cognitive therapy vs. psycho-education for patients with major depression who did not achieve remission following antidepressant treatment. Psychiatry Res. 2015;226(2-3):474-483. 
23. Manicavasgar V, Parker G, Perich T. Mindfulness-based cognitive therapy vs cognitive behaviour therapy as a treatment for non-melancholic depression. J Affect Disord. 2011;130(1-2):138-144.

24. Kazdin AE. Mediators and mechanisms of change in psychotherapy research. Annu Rev Clin Psychol. 2007;3(1):1-27.

25. van der Velden AM, Roepstorff A. Neural mechanisms of mindfulness meditation: bridging clinical and neuroscience investigations. Nat Rev Neurosci. 2015;16(7):439.

26. Gu J, Strauss C, Bond R, Cavanagh K. How do mindfulness-based cognitive therapy and mindfulness-based stress reduction improve mental health and wellbeing? A systematic review and meta-analysis of mediation studies. Clin Psychol Rev. 2015;37:1-12.

27. Alsubaie M, Abbott R, Dunn B, et al. Mechanisms of action in mindfulness-based cognitive therapy (MBCT) and mindfulness-based stress reduction (MBSR) in people with physical and/or psychological conditions: a systematic review. Clin Psychol Rev. 2017;55:74-91.

28. Batink T, Peeters F, Geschwind N, van Os J, Wichers M. How does MBCT for depression work? Studying cognitive and affective mediation pathways. PLoS One. 2013;8(8):e72778.

29. Kuyken W, Watkins E, Holden E, et al. How does mindfulness-based cognitive therapy work? Behav Res Ther. 2010;48(11):1105-1112.

30. Shahar B, Britton WB, Sbarra DA, Figueredo AJ, Bootzin RR. Mechanisms of change in mindfulness-based cognitive therapy for depression: Preliminary evidence from a randomized controlled trial. Int J Cogn Ther. 2010;3(4):402-418.

31. Bieling PJ, Hawley LL, Bloch RT, et al. Treatment-specific changes in decentering following mindfulness-based cognitive therapy versus antidepressant medication or placebo for prevention of depressive relapse. J Consult Clin Psychol. 2012;80(3):365-372.

32. Kearns NP, Shawyer F, Brooker JE, et al. Does rumination mediate the relationship between mindfulness and depressive relapse? Psychol Psychother. 2016;89(1):33-49.

33. Kabat-Zinn J. Wherever You Go, There You Are: Mindfulness Meditation in Everyday Life. New York: Hyperion; 1994.

34. Crane C, Winder R, Hargus E, Amarasinghe M, Barnhofer T. Effects of mindfulness-based cognitive therapy on specificity of life goals. Cogn Ther Res. 2012;36(3):182-189.

35. Britton WB, Shahar B, Szepsenwol O, Jacobs WJ. Mindfulness-based cognitive therapy improves emotional reactivity to social stress: results from a randomized controlled trial. Behav Ther. 2012;43(2):365-380.

36. Papageorgiou C, Wells A. Process and meta-cognitive dimensions of depressive and anxious thoughts and relationships with emotional intensity. Clin Psychol Psychother. 1999;6(2):156-162.

37. Fresco DM, Frankel AN, Mennin DS, Turk CL, Heimberg RG. Distinct and overlapping features of rumination and worry: The relationship of cognitive production to negative affective states. Cogn Ther Res. 2002; 26(2):179-188.
38. Nolen-Hoeksema S. Sex Differences in Depression. Vol vii. Stanford, CA: Stanford University Press; 1990.

39. Treynor W, Gonzalez R, Nolen-Hoeksema S. Rumination reconsidered: a psychometric analysis. Cogn Ther Res. 2003;27(3):247-259.

40. Geschwind N, Peeters F, Drukker M, van Os J, Wichers M. Mindfulness training increases momentary positive emotions and reward experience in adults vulnerable to depression: a randomized controlled trial. J Consult Clin Psychol. 2011;79(5):618-628.

41. Scher CD, Ingram RE, Segal ZV. Cognitive reactivity and vulnerability: Empirical evaluation of construct activation and cognitive diatheses in unipolar depression. Clin Psychol Rev. 2005;25(4):487-510.

42. Segal ZV, Williams JM, Teasdale JD. Mindfulness-Based Cognitive Therapy for Depression. New York: Guilford Press; 2012.

43. Williams JM, Penman D. Mindfulness: A Practical Guide to Finding Peace in a Frantic World. Hachette UK; 2011.

44. Lever Taylor B, Strauss C, Cavanagh K, Jones F. The effectiveness of self-help mindfulness-based cognitive therapy in a student sample: a randomised controlled trial. Behav Res Ther. 2014;63:63-69.

45. Felder J, Dimidjian S, Beck A, Boggs JM, Segal Z. Mindful mood balance: a case report of web-based treatment of residual depressive symptoms. Perm J. 2014;18(4):58-62.

46. Beck A, Dimidjian S, Boggs J, Felder J, Segal Z. PS2-43: Internet delivered mindfulness-based cognitive therapy for reducing residual depressive symptoms: an open trial and quasi-experimental comparison to propensity matched controls. Clin Med Res. 2014;12(1-2):104

47. Dimidjian S, Beck A, Felder JN, et al. Web-based mindfulness-based cognitive therapy for reducing residual depressive symptoms: an open trial and quasi-experimental comparison to propensity score matched controls. Behav Res Ther. 2014;63:83-89.

48. Parsons CE, Crane C, Parsons LJ, Fjorback LO, Kuyken W. Home practice in mindfulness-based cognitive therapy and mindfulness-based stress reduction: a systematic review and meta-analysis of participants' mindfulness practice and its association with outcomes. Behav Res Ther 2017;95:29-41.

49. Segal ZV, Williams JM, Teasdale JD, et al. Mindfulness-based cognitive therapy training pathway. Version 1.0. Oxford Mindfulness Centre; 2016. Available from: http://mbct.com/wp-content/uploads/MBCTTraining-Pathway-Final_Version1.pdf. Accessed May 24, 2018.

50. Crane RS, Eames C, Kuyken W, et al. Development and validation of the mindfulness-based interventions-teaching assessment criteria (MBI:TAC). Assessment. 2013;20(6):681-688.

51. Huijbers MJ, Crane RS, Kuyken W, et al. Teacher competence in mindfulness-based cognitive therapy for depression and its relation to treatment outcome. Mindfulness (N Y). 2017;8(4):960-972.

52. Lutz A, Jha AP, Dunne JD, Saron CD. Investigating the phenomenological matrix of mindfulness-related practices from a neurocognitive perspective. Am Psychol. 2015;70(7):632-658.
Neuropsychiatric Disease and Treatment

\section{Publish your work in this journal}

Neuropsychiatric Disease and Treatment is an international, peerreviewed journal of clinical therapeutics and pharmacology focusing on concise rapid reporting of clinical or pre-clinical studies on a range of neuropsychiatric and neurological disorders. This journa is indexed on PubMed Central, the 'PsycINFO' database and CAS,

\section{Dovepress}

and is the official journal of The International Neuropsychiatric Association (INA). The manuscript management system is completely online and includes a very quick and fair peer-review system, which is all easy to use. Visit http://www.dovepress.com/testimonials.php to read real quotes from published authors. 